\title{
Planning for the Next Generation of Health Care Delivery: Designing for Telemedicine Spaces
}

Robert Hume, PE, CCNA, CWNA, and Jeff Looney

The increase in telemedicine adoption has been driven by expanding reimbursements, the shortage of physicians due to the growing and aging population, the increase in insurance enrollment, changing health care delivery models, and technological innovations.

Telemedicine offers promise in reducing costs, increasing accessibility to care, and reducing the risk of healthcare-associated infections. Telehealth is explicitly listed as a strategy in three out of the four goals in the Health \& Human Services' Federal Health IT Strategic Plan for 2015-2020.

\section{Variety of Solutions}

The earliest telemedicine systems were fixed video teleconferencing (VTC) systems and, generally, still offer the highest quality video and audio. These are commonly used in conference rooms and education spaces. They consist of a camera, microphone, and hardware device called a codec (coder-decoder). Video is displayed on video monitors or projector screens. Popular manufacturers of VTC equipment include Polycom Inc., San Jose, Calif.; Cisco Systems Inc., San Jose; and Lifesize, a division of Logitech, Newark, Calif.

Telemedicine carts have become popular for bringing telemedicine services to any room. The hardware is essentially the same as a fixed VTC system but mounted to a cart for portability. Devices do not need to be duplicated (and potentially sit idle) in every telemedicine-capable space. A typical application would be stroke care in the emergency department, sometimes called "telestroke." Although telemedicine carts have many advantages, the need for staff to wheel the cart around may be 

troublesome. To overcome this, telemedicine vendor InTouch Technologies Inc., Santa Barbara, Calif., teamed up with iRobot Corp., Bedford, Mass., best known for the Roomba vacuum cleaning robot, to create RP-VITA, a remote presence telemedicine robot that promises to improve workflow efficiency by navigating between patient rooms (Figure 1).

While carts may be preferable in some cases, other telemedicine applications may be better served with fixed equipment in each patient room. In an electronic intensive care unit (eICU), remote intensivists are available $24 / 7$ to monitor patients in ICU patient rooms as support for on-site staff. An on-site caregiver may contact the remote intensivists using a wall-mounted call button on the headwall. The intensivists then can assess the patient using a pan-tilt-zoom camera and microphone at the footwall and communicate back to the ICU room via loudspeaker (and optionally a video monitor). The remote intensivists can do rounding and other forms of monitoring as well (Figure 2).

Most of the latest telemedicine solutions are Web-based systems running on standard computers or mobile devices. Services like Skype ${ }^{\mathrm{TM}}$, Google Hangouts and FaceTime brought video teleconferencing capability to the masses. While these products are easy to use and inexpensive, all are not appropriate for telemedicine. For example, Skype ${ }^{\mathrm{TM}}$ does not offer a business associate agreement for healthcare providers to meet HIPAA requirements.

There are alternatives to consumer video teleconferencing products for Web-based telemedicine. Companies offering business associate agreements for HIPAA compliance include Avizia Inc., Reston, Virginia; Blue Jeans Network ${ }^{\circledR}$, Mountain View, California; Aurora Information Technology, Cold Spring, New York; eVisit ${ }^{\circledR}$ Telehealth Solutions, Mesa, Arizona; SnapMD ${ }^{\circledR}$ Inc., Glendale, California; Vidyo ${ }^{\circledR}$, Hackensack, New Jersey; VSee Lab Inc., Sunnyvale, California; and Zoom Video Communications, San Jose, California. 

Web-based telemedicine solutions face challenges beyond HIPAA compliance. The American Medical Association states, "Whether a patient is seeing his or her physician in person or via telemedicine, the same standards of care must be maintained." Additionally, minimum technical requirements may need to be met. For example, American Telemedicine Association (ATA) guidelines require a minimum of $640 \times 360$ resolution and 30 frames per second for video. In other words, what's good enough for a chat with a friend or family member may not be good enough for telemedicine.

While communication with a camera and microphone is appropriate for many encounters, connected medical devices have the potential to make telemedicine much more powerful. For example AMD Global Telemedicine Inc., Chelmsford, Massachusetts, offers otoscopes, stethoscopes, dermascopes and ultrasound probes as well as vital signs monitors, spirometers and electrocardiography (ECG) equipment by Welch Allyn, Skaneateles Falls, New York, integrated with their telemedicine solution. The software allows split-screen viewing between the camera and medical devices. GlobalMedia Group LLC, Scottsdale, Arizona, offers the transportable exam station (TES), a rolling suitcase equipped with laptop, otoscope, stethoscope, ultrasound probe, ECG system and more. This is geared toward treating homebound and rural patients.

While some regional health facilities have arrangements with third parties or large hospitals to provide remote specialists for services like telestroke and elCU, larger health systems may provide their own specialists. A facility implementing a telemedicine program needs to determine how and where remote specialists will work. In October 2015, the Mercy health system in Saint Louis, Missouri, opened the Mercy Virtual Care Center-a 125,000-square-foot facility billed as the world's first virtual care center, to house specialists serving 28 Mercy sites. Mercy's telemedicine services include eICU, telestroke and home monitoring.

\section{Infrastructure Requirements}



The wide variety of telemedicine solutions has equally diverse infrastructure requirements. The impact includes the room location and size, flooring, lighting, power, data and conduit pathways. But the impact extends beyond a single room, requiring an assessment of network infrastructure, building services, power distribution and more. Even Web-based solutions using existing computers have potential impacts on a facility's design and physical infrastructure.

When planning for dedicated telemedicine rooms, they should be located in a quiet area away from loud mechanical equipment, foot traffic, the central utility plant and exterior noise sources. The Guidelines for Design and Construction of Hospitals and Outpatient Facilities, 2014 edition, published by the Facility Guidelines Institute, includes acoustics requirements for maximum noise levels, sound absorption coefficients, sound isolation between rooms and speech privacy, all of which must be considered for telemedicine.

Carpet is excellent at controlling sound, but finds limited use in clinical settings because of infection control concerns. Many facilities are turning to rubber flooring for both ease of cleaning and sound control. In fact, the new Parkland Memorial Hospital in Dallas, which opened in August 2015, includes more than 750,000 square feet of rubber flooring.

For telemedicine encounters occurring in an exam room, equipment placement must be considered, with adequate space provided for the telemedicine cart and associated staff members. The room also must be large enough to position the cart-mounted camera with an appropriate view of the patient and staff.

The backdrop of the video should be free of distractions and clutter. Some facilities place signage with the site location in the background to help orient participants. The choice of paint is also important. Generally, white walls and dark walls should be avoided. A light neutral color is preferable. Powder blue is often recommended to provide contrast with flesh tones. Flat or matte paint is preferable to glossy paint to reduce glare. 
Lighting cannot be overlooked for telemedicine video. Overhead fluorescent lights alone are often insufficient, requiring additional lighting. The best lighting is diffuse light in front of the subject since it provides even illumination without strong shadows. Windows should be avoided, especially behind the subject, because bright sunlight may wash out the video image or create extreme shadows. Telemedicine devices, such as otoscopes, should be used with a light source.

The VTC equipment itself also will impact infrastructure. A fixed VTC system typically is installed with conduit pathways for cabling from the microphones, cameras and monitors back to the codec. The codec may be installed on a shelf or in a rack, credenza or podium. The codec requires power and network connections. Audiovisual $(A / V)$ equipment enabling routing of sources to displays is usually provided in conference rooms in addition to the VTC equipment, because these rooms serve multiple functions. Telemedicine carts eliminate the need for most in-wall A/V cabling, but power outlets and network connections must be provided where the cart will be in use. A telephone should be provided in telemedicine spaces as a backup means of communication as well as for troubleshooting the VTC system.

Camera placement is crucial for conference rooms and offices. The camera should be at eye level and a few feet from the subject to mimic face-to-face encounters. Microphone selection and placement is equally important to ensure clear speech communication. For offices, a headset microphone ensures consistent sound levels and minimal echo and noise. For conference rooms, table-mounted microphones evenly pick up voices around the table, though they may require holes drilled into the table, wiring, and a floor box with conduit pathway. Ceiling-mounted microphones may be a better option to reduce infrastructure impact or if the conference table is movable.

\section{elCU Considerations}



An eICU also requires significant physical infrastructure. Typically, each patient room requires an $\mathrm{A} / \mathrm{V}$ server in an enclosure, which requires a power outlet and a network connection. The camera, microphone, speaker and call button are wired back to the A/V server, preferably through conduit pathways. The camera also may require a power outlet. The camera, speaker and monitor need to be wall- or ceiling-mounted. The placement of the call button is important and requires clinician input. Even if a singlegang, low-voltage provision is free on a headwall system, there may not be sufficient clearance for the call button. The call button should be no more than 48 inches above the finished floor to meet Americans with Disabilities Act (ADA) requirements. A telephone in the patient room that directly dials the eICU remote intensivists is required as a backup means of communication. The eICU system also requires rack space for servers in the data center.

Facilities dedicated to telemedicine are a recent development, and best practices for design have not yet been established. The design of Mercy Virtual Care Center focused on large, open work areas. These open work areas are intended to increase collaboration, as well as provide flexibility for future changes. To offset the long hours caregivers spend in front of computers, floor-to-ceiling glass walls provide views of nature. Privacy screens placed behind the caregivers serve the dual purpose of protecting patient privacy and serving as the video backdrop.

Equipment used for telemedicine, including computers, monitors, VTC equipment and carts, should be powered by an uninterruptible power supply (UPS) connected to critical power, if possible. Network connections should be wired rather than wireless. Even if the wireless network can support a telemedicine session, the traffic will negatively impact all other devices connected to the wireless network nearby.

Because telemedicine solutions likely use the hospital's local area network (LAN), the network must have high availability. A UPS connected to critical power should power network equipment. Additionally, each network switch may have redundant power supplies, which protect against internal power supply failures and allow separate 

circuits, or even separate power feeds, to provide redundant power. Redundant fiber links may be provided between telecommunications rooms and the network core to protect against the failure of a single link. Links may take separate pathways for additional protection.

Services to the building may be redundant as well. Separate service pathways are even a requirement in National Fire Protection Association (NFPA) 99, Health Facilities Code, $\underline{2012}$ and $\underline{2015}$ editions. In the past, video teleconferencing systems used integrated services digital network (ISDN) lines dedicated to the VTC system. Most systems now use Internet Protocol (IP) with the VTC traffic sharing the facility's connection to the Internet service provider. Because this arrangement lacks the predictable bandwidth of dedicated lines, network Quality of Service configurations are important.

The bandwidth required for a video teleconference varies depending on the resolution, frame rate and video encoding. ATA guidelines call for a minimum of 384 kilobits per second (kbps) bidirectional. High-definition video teleconferencing typically requires between $768 \mathrm{kbps}$ and $1024 \mathrm{kbps}$. Because most LAN ports are 100 megabits per second or 1 gigabit per second, data rates from the service provider, not the LAN itself, are the most likely bottleneck. This is especially true if multiple video teleconferences occur simultaneously in the same facility.

\section{Considering the Challenges}

While telemedicine must overcome additional policy and reimbursement hurdles to become widely adopted, it will continue to grow in the coming years. Facilities must look at telemedicine comprehensively and consider the operational, financial, ethical, and legal challenges.

Once those issues have been addressed, a facility must understand the technology and infrastructure repercussions for their telemedicine implementation. These infrastructure impacts are far-reaching, but facilities professionals cannot ignore them. 
Article type: Perspective, opinion, interview, and commentary Tags: health care, healthcare, infrastructure, platform, challenges, teleconference, technological, innovations, patients, teleconferencing, HIPAA, homebound, rural

Robert Hume, PE, CCNA, is a project manager and Jeff Looney is vice president at GBA, a health care technology-consulting firm based in Franklin, Tennessee. They can be reached at Robert.Hume@gbainc.com and Jeff.Looney@gbainc.com, respectively.

This article is reprinted with permission of Health Facilities Management.

http://www.hfmmagazine.com/display/HFM-news-

article.dhtml?dcrPath=/templatedata/HF Common/NewsArticle/data/HFM/Magazine/20 $\underline{16 / f e b / h f m-t e l e m e d i c i n e-a n d-f a c i l i t y-d e s i g n ~}$

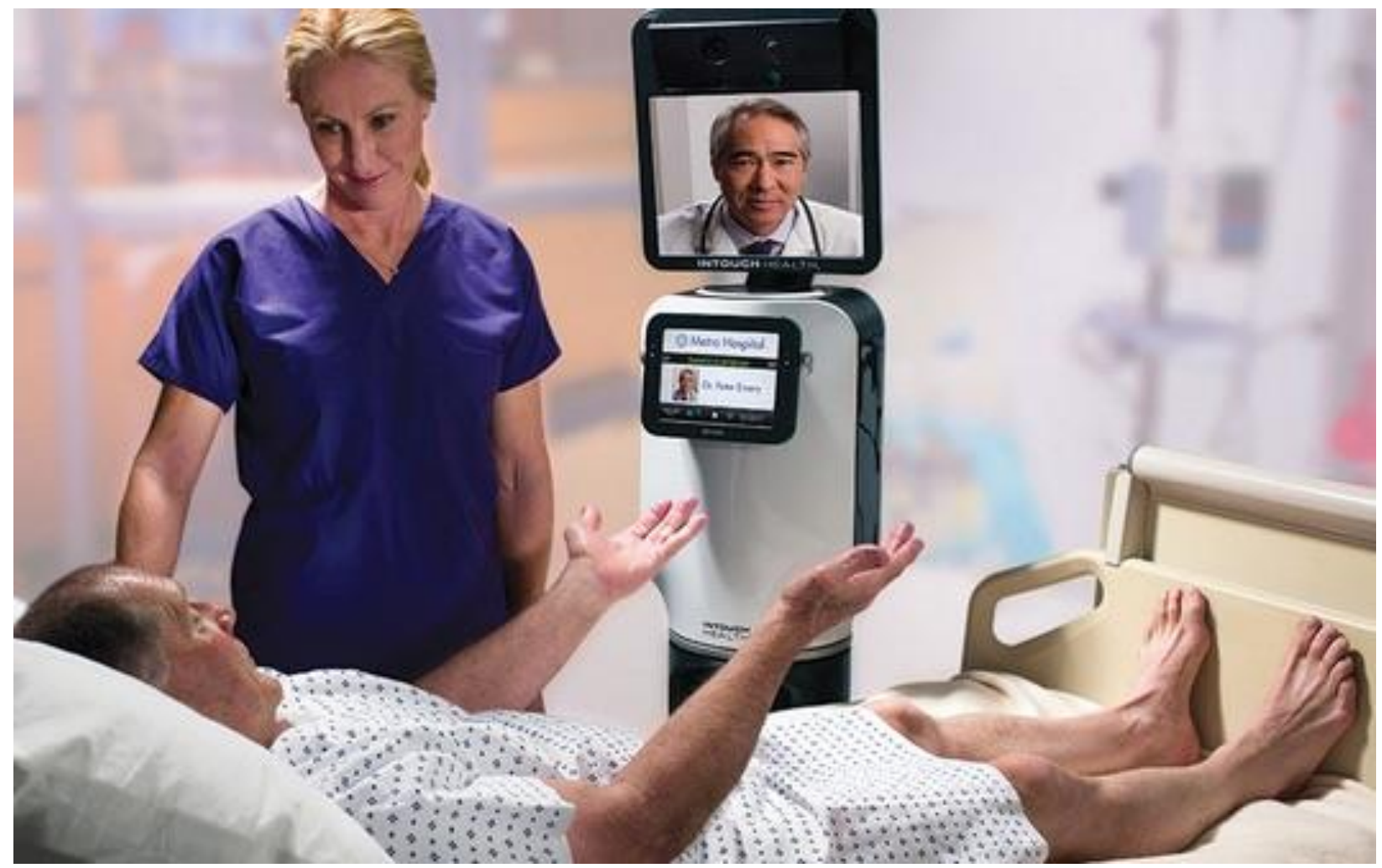

Figure 1. For telemedicine encounters occurring in an exam room, equipment placement must be considered with adequate space provided for the telemedicine cart 


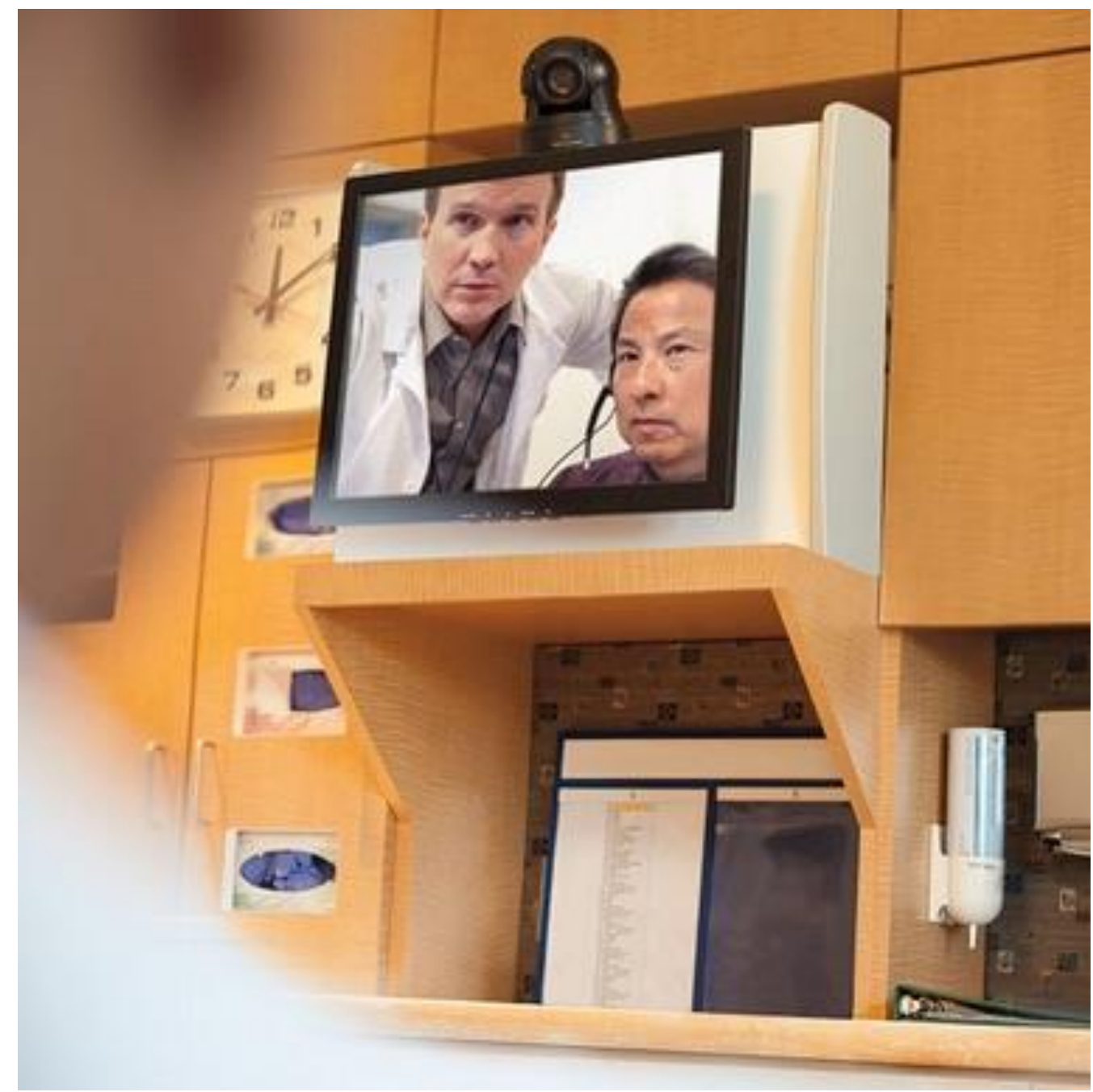

Figure 2. In an electronic intensive care unit (elCU), remote intensivists are available 24/7 to monitor patients in ICU patient rooms as support for on-site staff. Photo courtesy of Philips. 